\title{
Light Electrospun Polyvinylpyrrolidone Blanket for Low Frequencies Sound Absorption
}

\author{
Joshua Avossa ${ }^{\mathrm{a}}$, Francesco Branda ${ }^{\mathrm{a}}$, Francesco Marulo ${ }^{\mathrm{b}}$, Giuseppe Petrone ${ }^{\mathrm{b}}$, Stefano Guido ${ }^{\mathrm{a}}$, \\ Giovanna Tomaiuolo ${ }^{a}$, and Aniello Costantini ${ }^{\mathrm{a}}$ \\ ${ }^{a}$ Dipartimento di Ingegneria Chimica, dei Materiali e della Produzione Industriale (DICMaPI), Università degli Studi di Napoli Federico II, Napoli, Italy \\ b Dipartimento di Ingegneria Industriale (DII), Università degli Studi di Napoli Federico II, Napoli, Italy
}

\begin{abstract}
Light polymeric soundproofing materials (density $=63 \mathrm{~kg} / \mathrm{m}^{3}$ ) of interest for the transportation industry were fabricated through electrospinning. Blankets of electrospun polyvinylpyrrolidone (average fiber diameter $=(1.6 \pm 0.5)$ or $(2.8 \pm 0.5) \mu \mathrm{m})$ were obtained by stacking disks of electrospun mats. The sound absorption coefficients were measured using the impedance tube instrument based on ASTM E1050 and ISO 10534-2. For a given set of disks (from a minimum of 6) the sound absorption coefficient changed with the frequency (in the range 200-1600 Hz) following a bell shape curve with a maximum (where the coefficient is greater than 0.9) that shifts to lower frequencies at higher piled disks number and greater fiber diameter. This work showed that electrospinning produced sound absorbers with reduced thickness $(2-3 \mathrm{~cm})$ and excellent sound-absorption properties in the low and medium frequency range.
\end{abstract}

Keywords Electrospinning; Polyvinylpyrrolidone (PVP); Sound absorption; Impedance tube

Citation: Avossa, J.; Branda, F.; Marulo, F.; Petrone, G.; Guido, S.; Tomaiuolo, G.; Costantini, A. Light Electrospun Polyvinylpyrrolidone Blanket for Low Frequencies Sound Absorption. Chinese J. Polym. Sci. 2018, 36(12), 1368-1374.

\section{INTRODUCTION}

Noise pollution is one of the most widespread problems in modern society and constitutes real danger to the human health ${ }^{[1-4]}$. The potential health effects of noise pollution are numerous, pervasive, persistent, and medically and socially significant. Noise produces direct and cumulative adverse effects that impair health and that degrade residential, social, working, and learning environments with corresponding real (economic) and intangible (well being) losses ${ }^{[5]}$.

For this reason, the production of materials for noise control has increased as a result of technological changes and product requirements in many areas, including buildings, aircraft and spacecraft, ground and marine transportation vehicles, and manufacturing facilities. In particular, the reduction of interior noise levels in the transportation industries remains a big problem to cope with[2, 3, 6-9] nowadays by the strong interest devoted to the passengers' comfort.

Commercial sound absorber materials that have high values of the sound absorption coefficient are usually porous ${ }^{[9]}$. However they exhibit good sound absorption properties in the high frequency range but little in the low and medium frequency range $(250-1600 \mathrm{~Hz})$, in which human sensitivity is high ${ }^{[9]}$.

\footnotetext{
* Corresponding author: E-mail branda@unina.it

Received March 16, 2018; Accepted May 8, 2018; Published online July 11, 2018
}

Innovative polymeric sound absorbers can be produced through electrospinning. Such technique can be used to produce micro/nanofibers ranging from $2 \mathrm{~nm}$ to several micrometers ${ }^{[10-16]}$, in contrast to conventional synthetic fiber forming processes such as those used for high speed spinning of nylon or polyester, where continuous fibers ranging from $10 \mu \mathrm{m}$ to $500 \mu \mathrm{m}$ are produced. Electrospinning has already been proposed in the field of sound absorption thanks to the small diameter of the electrospun fibers. The electrospun materials possess high specific surface area 100 to 10000 times greater than that of the traditional acoustical fibrous materials; as a consequence, noise absorption is expected to rise due to the higher friction between the air molecules of sound waves and the electrospun fibers and/or rubbing of the fibers $^{[3]}$. Several reports on the acoustical performance of electrospun polymer fibers well confirm the interest in the production of sound-absorbing materials through electrospinning $[3,10,17,18]$. Xiang et al. used micrometer electrospun membranes of polyacrylonitrile combined with traditional acoustical materials (perforated panel, foam and fibers material), and the sound-absorbing efficiency can be greatly enhanced especially in the low and medium frequency range[17]. In a similar work, Trematerra et al. showed that millimeter electrospun layers can enhance sound absorption of conventional sound absorber materials (glass wool, kenaf, foam and polyester with thickness of 3 and $4 \mathrm{~cm})^{[18]}$. Khan et al. combined different electrospun mats (polyvinylpyrrolidone, polystyrene and polyvinylchloride) with fibers of diameter ranging from $0.3 \mu \mathrm{m}$ to 
$7 \mu \mathrm{m}$. The best absorbing properties were obtained by the combination of electrospun PVP and PS layers ${ }^{[3]}$. So far, the electrospun materials alone ${ }^{[3]}$ or coupled with other materials $^{[17,18]}$ enabled great absorption after $1000 \mathrm{~Hz}$. Nevertheless, such materials did not show efficient sound absorption in range of low frequencies $(<1000 \mathrm{~Hz})$ that are much more difficult to absorb than the medium and high frequency range sounds. Thus, the challenges to the present state in the transportation sector is to produce low weight and/or reduced thickness sound absorbers for the low and medium frequency range sounds $(250-1600 \mathrm{~Hz})$.

In the present work, sound-absorbing blankets were successfully produced by stacking thin disks of light nonwoven mats $(10 \mathrm{~cm}$ diameter, $\max 0.700 \mathrm{~g}$ in weight) on each other until a maximum thickness of $2.5 \mathrm{~cm}$ was obtained by electrospinning PVP of high molecular weight $(1300 \mathrm{kDa})$. We obtained a very high sound absorption coefficient value $>0.9$ at $\sim 450 \mathrm{~Hz}$ which is very interesting compared to the previous works $[3,17,18]$. Such sound absorption coefficient values at low frequencies range are reported $^{[9]}$ for micro-perforated panels (MPP), with the technological problem, however, of producing thin panels having hundreds of sub-millimeter perforations per square centimeters $^{[9]}$ or for some glass wool mats used in the aerospace industry.

\section{EXPERIMENTAL}

\section{Materials}

Polyvinylpyrrolidone with a molar mass of $1.30 \times 10^{6} \mathrm{~g} / \mathrm{mol}$ and ethanol with a purity $\geq 99.8 \%$ were purchased from Sigma-Aldrich and used without any additional purification or processing.

\section{Preparation of Electrospun Layer}

The electrospinning apparatus used in this work, whose scheme, in a horizontal set up, shown in Fig. 1, consists of a high voltage electric source, a syringe pump (Harvard Apparatus, Pump 11 Plus) holding a $12 \mathrm{~mL}$ plastic syringe (Nipro) with a needle (inner diameter of $0.6 \mathrm{~mm}$ and average length of $4 \mathrm{~cm}$ ) acting as the cathode and a rigid copper foil collector acting as the anode.

Two series of samples, in the form of thin disks of mass approximately equal to $0.5 \mathrm{~g}$, were produced from

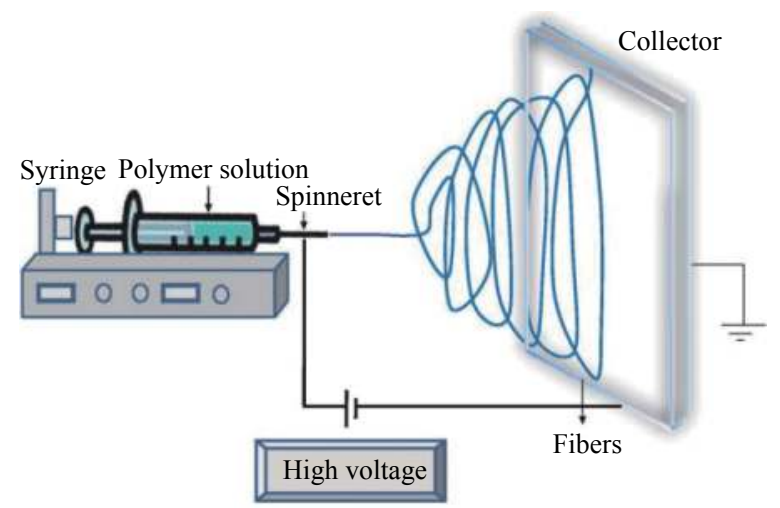

Fig. 1 Scheme of an electrospinning apparatus in horizontal set up
PVP/ethanol solutions with different polymer concentrations. Each disk will be distinguished, in the following, with a symbol $\beta_{i}$ or $\delta_{i}$. The first one indicates the $i$-th disk of series obtained from solutions $0.10 \mathrm{~g} / \mathrm{mL}$ of PVP in ethanol ( $\beta$ series). In the other case ( $\delta$ series) the concentration was higher: $0.15 \mathrm{~g} / \mathrm{mL}$. In all the cases a flow rate of $0.200 \mathrm{~mL} / \mathrm{min}$ was assured by the syringe pump; the solutions were electrospun under an applied electrical potential of $20 \mathrm{kV}$ over a fixed collection distance of $39 \mathrm{~cm}$ at room temperature $\left((23 \pm 2){ }^{\circ} \mathrm{C}\right)$ and relative humidity $((45 \pm 10) \%)$. To convey the electrospinning jet onto the collector, a copper string was placed at the bottom of the chamber at the same potential of the needle nozzle. The as-prepared electrospun non-woven mats were dried out at $100^{\circ} \mathrm{C}$ for $90 \mathrm{~min}$ and stored in a desiccator.

\section{Characterization}

Scanning electron microscopy (SEM)

Small cuts of the PVP electrospun discs were used to conduct scanning electron microscopy (SEM, FEI Inspect) observations. The samples were placed horizontally on an adhesive support and then coated by a thin layer of gold or carbon by plasma-assisted deposition. After that, the sample support was placed into the vacuum chamber of the microscope for image acquisition process. The morphology and average diameter of the fibers were characterized by off line image analysis techniques of the acquired images (Image-Pro Plus). The statistical analysis was performed by measuring the diameters of hundreds of fibers of ten different micrographs.

Flow resistivity

As described elsewhere ${ }^{[19]}$ the air flow resistivity was measured according to EN 29053-ISO 9053 through the experimental device SCS 9023 of SCS Controlli e Sistemi s.r.l., with the alternate airflow method formerly proposed by Whole and Weber based on a sinusoidal airflow at the frequency of $2 \mathrm{~Hz}$. The reported results are the average of three values obtained on three different samples in the shape of discs of $10 \mathrm{~cm}$ diameter and $1 \mathrm{~cm}$ thickness.

Sound absorption coefficient

The goal of the sound measurement was to determine the normal incidence sound absorption coefficient (ratio between the energy absorbed by the material and the incident energy of the sound wave) as a function of sound frequency. An acoustic impedance tube was used to test material samples for normal incidence sound absorption according to ASTM E1050 (standard test method for impedance and absorption of acoustical materials using a tube, two microphones and a digital frequency analysis system) and ISO 10534-2 (determination of sound absorption coefficient and impedance in an impedance tube-part II: transfer function method). The functioning was continuously checked with a standard calibrated sample. Fig. 2 shows the measurement setup in the laboratory, the impedance tube and Pulse LabShop used in this study. The two-microphone transfer function method was used according to ASTM E 1050-12 and and ISO 10534-2. In the impedance tube, a loudspeaker is mounted at one end and a material sample is placed at the other end. The loudspeaker generates sound waves that 

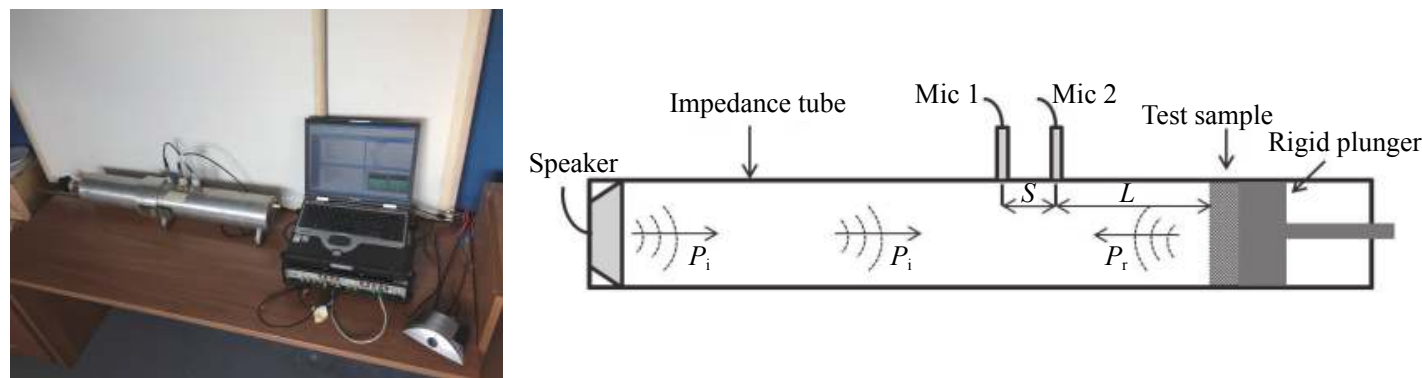

Fig. 2 Acoustic sound absorption coefficient measurement: measurement setup in the laboratory (left) and schematic of two-microphone impedance tube method (right)

propagate as plane waves in the tube and are reflected off the sample surface (Fig. 2). This leads to a standing-wave interference pattern resulting from forward and backwardtravelling waves inside the tube. The sound pressure is measured at two microphone fixed locations. The complex sound reflection coefficient $R$ of a tested sample is calculated from the corrected acoustic transfer function $H_{12}$. According to Chung and Blaser's ${ }^{[20]}$ results, the complex sound reflection coefficient is:

$$
R=R_{\mathrm{r}}+j R_{\mathrm{i}}=\frac{H_{12}-\mathrm{e}^{-j k S}}{\mathrm{e}^{j k S}-H_{12}} \mathrm{e}^{2 j k(S+L)}
$$

where $k=2 \Pi f / c$ is the (real) wave number in the air, $c$ is the sound speed in the air, $f$ is the working frequency and $j=$ $\sqrt{-1}$. From Eq. (1) it is possible to calculate the sound absorption coefficient at normal incidence as a function of frequency ${ }^{[20,21]}$ and the normal acoustic specific impedance respectively as:

$$
\begin{aligned}
\alpha & =1-|R|^{2}=1-R_{\mathrm{r}}^{2}-R_{\mathrm{i}}^{2} \\
\frac{Z}{Z_{0}} & =\frac{r}{\rho_{0} c_{0}}+\frac{j x}{\rho_{0} c_{0}}=\left(\frac{1+R}{1-R}\right)
\end{aligned}
$$

where $Z_{0}=\rho_{0} c_{0}$ is the characteristic impedance of the medium with $\rho_{0}$ and $c_{0}$ respectively density and speed of sound of the air, $r /\left(\rho_{0} c_{0}\right)$ is the normal specific acoustic resistance ratio and $x /\left(\rho_{0} c_{0}\right)$ is the normal specific acoustic reactance ratio. The normal sound absorption coefficient indicates the ability of the porous material to absorb sound energy in different frequency bands. The valid frequency range depends on the diameter of the tube and the spacing between the microphone positions. According to the geometry of the used instrument, sound absorber materials with a diameter of $10 \mathrm{~cm}$ can be tested in the range 200
$1600 \mathrm{~Hz}$. The normal incidence surface impedance, indeed, is a complex coefficient given by the acoustical pressure to velocity ratio at the surface of the tested sample when it is excited by a normal incidence acoustical wave. It measures the resistance and inertia encountered by the acoustical wave trying to penetrate the material. Its real part is the acoustical resistance and its imaginary part is the acoustical reactance.

\section{RESULTS AND DISCUSSION}

\section{Electrospun Mats Structure}

The electrospun mats were produced as thin discs with a diameter of $10 \mathrm{~cm}$ from high molecular weight $(1300 \mathrm{kDa})$ PVP solutions in ethanol. The disks were cut from slightly larger electrospun deposits obtained in the conditions described in the experimental section. So as described in the experimental section the disks were produced and distinguished with an acronym $\beta_{i}$ or $\delta_{i}$. The acronym $\beta_{i-j}$ or $\delta_{i-j}$ indicate a pile made by stacking all the disks from the $i$-th to $j$-th one.

In Figs. 3(a)-3(c) the SEM micrographs taken on the $\beta_{18}$ disk, representative of all other $\beta$ ones, are reported. As can be seen, the mats have a general fibrous morphology (Fig. 3a) even if a few beads-on-string are present (Fig. 3b). Fig. 3(c) shows that the surface of fibers and beads is quite rough. The fiber average diameter is equal to $1.6 \mu \mathrm{m}$ with a standard deviation of $0.5 \mu \mathrm{m}$. No difference in fiber diameter is observed between the two opposite faces of the disk. This structural homogeneity may be the result of producing thin disks (10 cm diameter, max $0.700 \mathrm{~g}$ in weight) to be piled on each other. In fact slight progressive alteration of the electric field profile, due to the progressive deposition of the fibers on the target, may affect the electrospinning process ${ }^{[12]}$, leading to potential structural variations during electro-
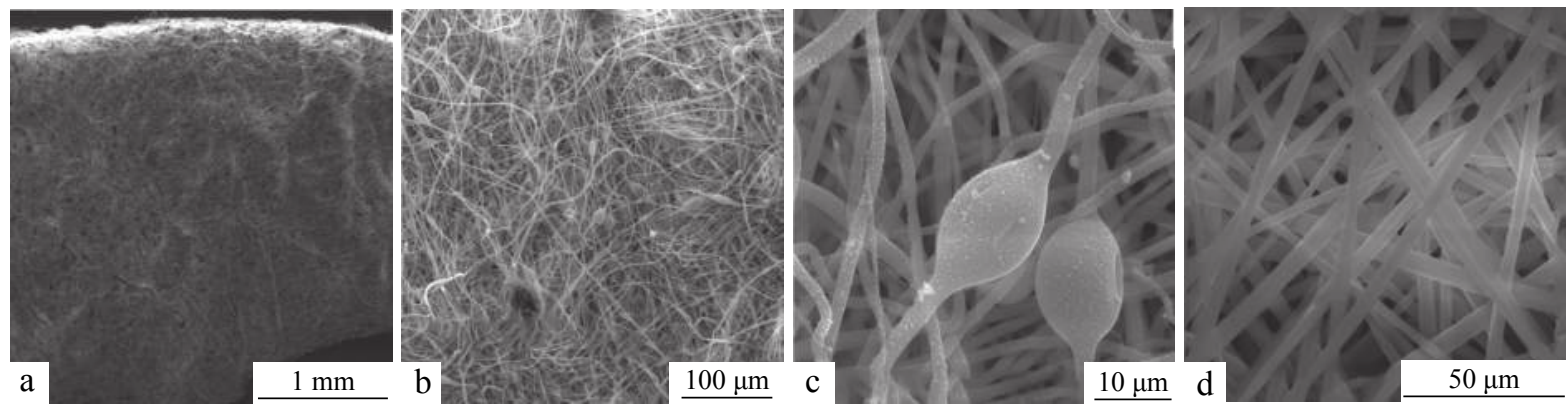

Fig. 3 SEM images of $\beta(\mathrm{a}-\mathrm{c})$ and $\delta(\mathrm{d})$ PVP electrospun disks 
spinning of overly thick mats. Fig. 3(d) shows the high magnification SEM micrograph of sample $\delta 10$ representative of all other $\delta$ ones. The lower magnification micrographs are similar to the ones (Figs. $3 \mathrm{a}$ and $3 \mathrm{~b}$ ) of $\beta 18$ disk but the differences that are better seen in Fig 3(d) are the absence of beads and a larger mean diameter, $(2.8 \pm 0.5) \mu \mathrm{m}$.

As it is known, many parameters affect the electrospinning process, which are well described in the literature ${ }^{[11-14,16]}$. It is worth remembering that the polymer molecular weight is one of the most important parameters[11-14, 16, 22]. In particular: (1) the greater the molecular weight, the lower the polymer concentration that assures the occurrence of sufficient chain entanglements and, therefore, the avoidance of bead formation ${ }^{22]}$; (2) the fiber size increases ${ }^{[13,14,16,22]}$ with increasing molecular weight and polymer concentration in the electrospun solution.

Therefore the absence of beads and the greater fiber diameter may be due to the greater PVP concentration of $\delta$ sample electrospun solution. This may also explain the differences in the experimental conditions that had to be used in the present work, and in the final structure of the present electrospun mats with respect to the ones possessing good acoustical properties, recently described by other authors ${ }^{[3]}$. The use of PVP of high molecular weight $1300 \mathrm{kDa}$, instead of $130 \mathrm{kDa}^{[3]}$ of the previous work, required a much lower concentration of the electrospun polymer: $10 \%-15 \%$ in ethanol instead of $80 \%$ of the previous work. Moreover, the fiber diameter was $(1.6 \pm 0.5)$ or $(2.8 \pm 0.5) \mu \mathrm{m}$ instead of $750 \mathrm{~nm}$ of the previous work.

\section{Electrospun Mats Acoustic Properties}

The disks were submitted to the acoustic impedance tube analysis for the measurement of the absorption coefficient in the frequency range $200-1600 \mathrm{~Hz}$, where the performance of traditional porous materials is usually poor. The normal incidence sound absorption coefficient was measured as a function of sound frequency.

First, the analysis was performed on one disk and then repeated after the addition of one more disk at a time. Before each analysis, a pressure was exerted through a metal disk of $130 \mathrm{~g}$ for one minute, to be sure that the disks adhered to each other in the pile and to completely eliminate the air layer that could remain entrapped between the electrospun disks when put on each other. No connection was established between the disks - after the measurements they could be separated and reassembled in a different order.

The pile of disks was simply leant against the bottom of the acoustic impedance tube. Each measurement was repeated 3-5 times (the test sample was removed and reinserted in the impedance tube each time) and the final result was an average of the repeated measurements. Fig. 4 shows the photograph of the final pile of $\beta$ discs prepared for the acoustic impedance tube. It reached a height of $2.5 \mathrm{~cm}$. A rough estimate of its volume allowed to make a rough evaluation of the apparent density equal to $63 \mathrm{~kg} / \mathrm{m}^{3}$. A very close value, $64 \mathrm{~kg} / \mathrm{m}^{3}$, was measured for the $\delta$ discs. It is worth pointing out that this value is very close to the ones reported for the very popular stone and glass wool largely used for the thermal and acoustical insulation in civil engineering applications. Fig. 5 shows some representative results of acoustic impedance tube analysis for $\beta$ samples in the form of the plot of absorption coefficient, $\alpha$, as a function of the frequency.

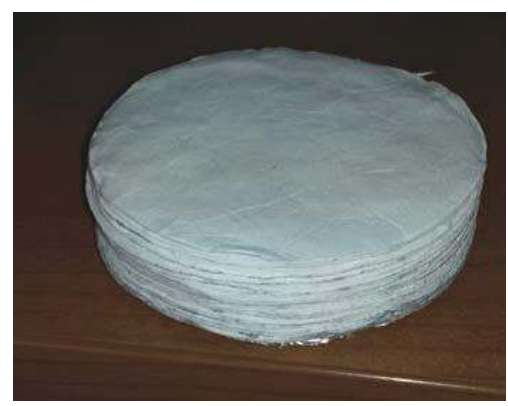

Fig. 4 Photograph of the pile of 22 disks

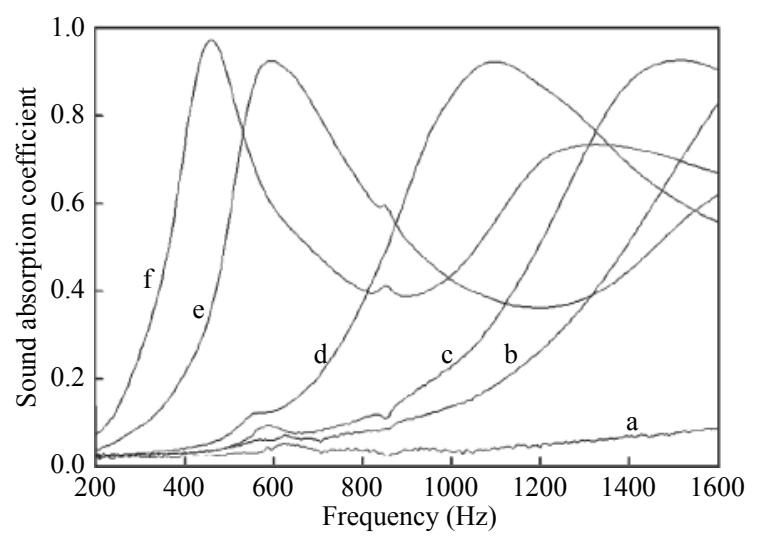

Fig. 5 Plots of the sound absorption coefficient as a function of frequency for various $\beta$ disks piles. The $\mathrm{a}, \mathrm{b}, \mathrm{c}, \mathrm{d}$, e and $\mathrm{f}$ curves refer, respectively, to the samples $\beta_{1}, \beta_{1-4}, \beta_{1-6}, \beta_{1-10}, \beta_{1-17}$ and $\beta_{1-22}$, ( $\beta_{i-j}$ means the pile made of all the disks from the $i$-th to $j$-th).

The absorption coefficient of the first disk $\beta_{1}$ (curve a) was very low in the examined frequency range. $\alpha$ values increased when the disks were stacked on each other. As soon as 6 disks were piled up (see curve c), it appeared that the acoustical response of the samples could be represented by a bell shape curve with a maximum $\alpha>0.9$. When the analysis was repeated changing the identity of the disks, no differences were observed provided that the total mass was the same-it appeared that the result was related to the total mass, not to the number nor the specific identity of the piled disks. For example, when measuring the absorption coefficient of two different piles, sample $\beta_{6-12}$ and $\beta 18-21$, having the same total mass as the $\beta_{1-4}$ one (composed of the first 4 disks) the same curve reported as curve $b$ (relative to the $\beta$ 1-4 pile) was obtained as can be seen in Fig. 6. It is worth observing that the curves of Fig. 5 shift towards lower frequency and become sharper with greater number (and total mass) of the disks. Fig. 5 shows only a part of the results. Indeed the measurement was repeated by progressively adding one disk at a time to the pile. Fig. 7 shows that similar results were obtained for $\delta$ samples.

It is worth underlining a remarkable result: very high sound absorption coefficients were measured. Comparable 


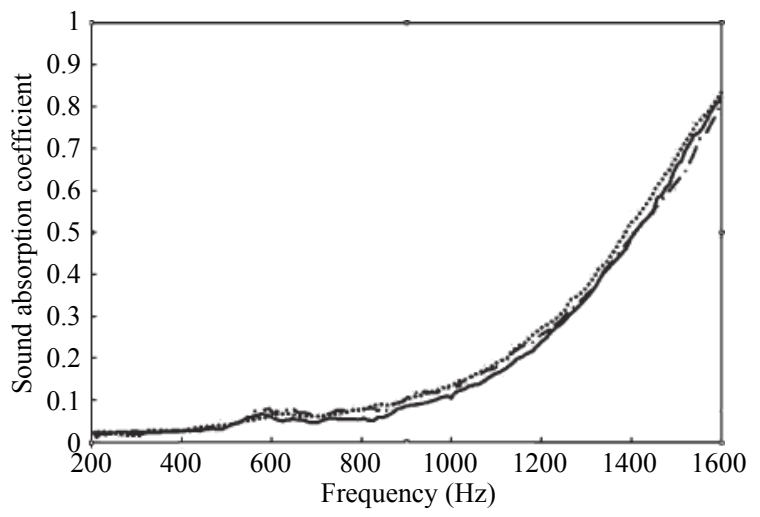

Fig. 6 Plots of the sound absorption coefficient as a function of frequency for $\beta_{1-4}(\ldots \ldots), \beta_{6-12}(---)$ and $\beta_{18-21}$ (---) disk piles having the same total mass

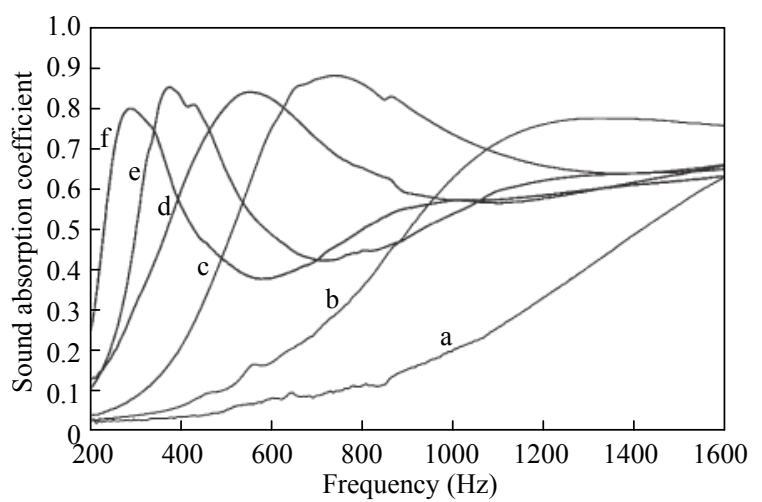

Fig. 7 Plots of the sound absorption coefficient as a function of frequency for various $\delta$ disks piles. The a, b, c, d, e and f curves refer, respectively, to the samples $\delta_{1-3}, \delta_{1-6}, \delta_{1-10}, \delta_{1-14}, \delta_{1-20}$ and $\delta_{1-26}$ ( $\delta_{i-j}$ means the pile made of all the disks from the $i$-th to $j$-th).

values of the absorption coefficient, in the $800-1000 \mathrm{~Hz}$ frequency range, were reported ${ }^{[9]}$ for heavier and/or thicker samples. This is confirmed by Figs. 8 and 9 where the sound absorption curves of electrospun PVP and glass wool samples marketed in civil and aerospace engineering fields are compared. In Fig. 8, the comparison is made between samples of similar mass per unit surface. In Fig. 9 the results for samples of similar thickness are compared. Fig. 8 shows that the PVP samples may have better sound absorption properties in the lower frequency range than materials of the same mass that are usually traded in the field of civil engineering. However PVP samples may have better sound absorption properties in the lower frequency range than materials, of same thickness, usually traded in both civil and aerospace engineering fields.

Figs. 5 and 7 show that when the disk pile was composed of a proper number of disks ( 6 for the $\beta$ series), $\alpha$ changed with the frequency following a bell shape curve. A tunable effect was also observed - the frequency at which $\alpha$ reached the maximum value $(>0.9)$ changed with the number (and therefore the mass) of the disks constituting the pile. A second relative maximum was observed for some curves (curves e and $\mathrm{f}$ in Fig. 5 for $\beta$ series and curves e and $\mathrm{f}$ in Fig. 7 for $\delta$ series). The second maximum of curves occured at a frequency about 3 times greater than the one of the first

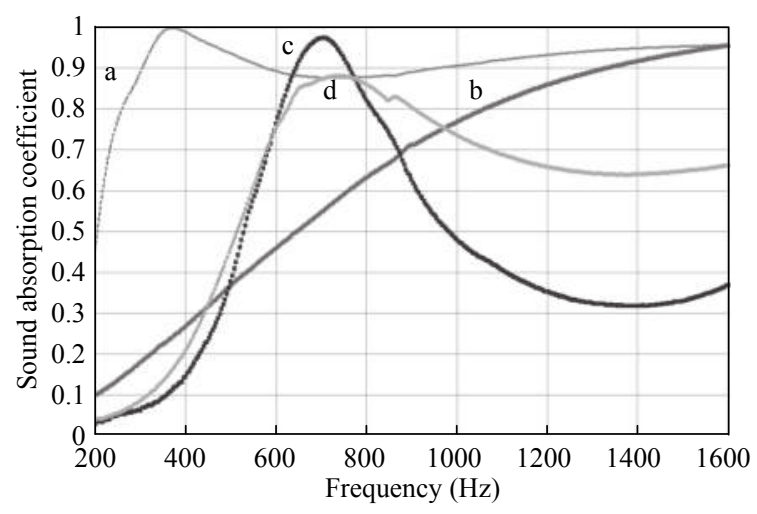

Fig. 8 Sound absorption coefficients of samples of same mass per unit surface $\left(0.89 \mathrm{~kg} / \mathrm{m}^{2}\right)$ of PVP beta (c) and delta (d) and glass wool samples of civil (b) and aerospace (a) engineering fields

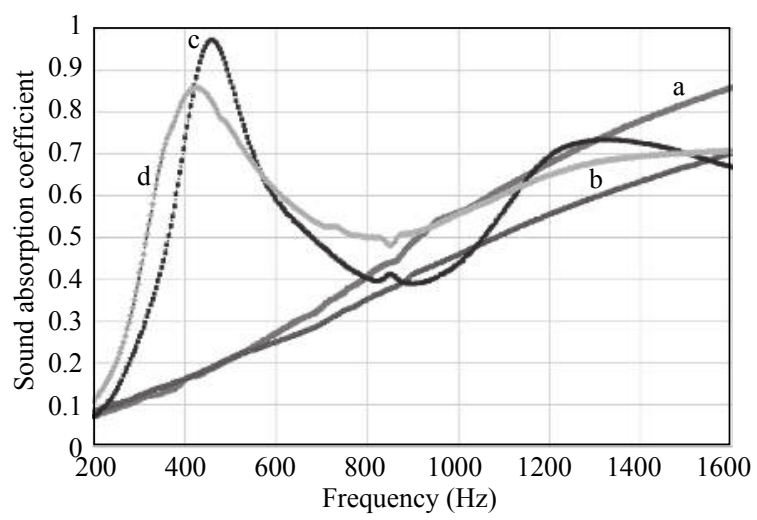

Fig. 9 Sound absorption coefficients of samples of same thickness $(2.54 \mathrm{~cm})$ of PVP beta (c) and delta (d) and glass wool samples of civil (b) and aerospace (a) engineering fields

maximum. In the case of curve e in Fig. 5 the presence of a second maximum may be imagined even if only the growing part of it is seen. The curves for lower masses might be shifted too much towards higher frequencies to allow observing the second maximum.

The frequency for which $\alpha$ reached the maximum value was determined for each curve starting from the sample $\beta$ 1-6 and $\delta_{1-6}$. It is reported in Fig. 10 as a function of the mass per unit surface of the disks progressively stacked on each other. Fig. 10 shows that the shift of the bell like curves towards lower frequencies can be continuously tuned by changing the mass of the blanket. Taking into account that the two mats differ for the fiber diameter, Fig. 10 suggests that the fiber radius increase made the frequency of maximum sound absorption decrease.

The flow resistivity of the samples was measured according to EN 29053-ISO 9053. The values are reported in Table 1. In the same table the values of fiber diameter and density evaluated as described above, from the SEM micrographs and mass and weight of blankets, are reported. The flow resistivity value for the $\beta$ sample is higher than the one for sample $\delta$. It is worth observing that both values are much greater than the ones $\left(1-40 \mathrm{kPa} \cdot \mathrm{s} / \mathrm{m}^{2}\right)$ reported for glass wool[23, 24]

In order to explain the results it is worth reminding $[1-4,9]$ that sound energy can be absorbed by the following 


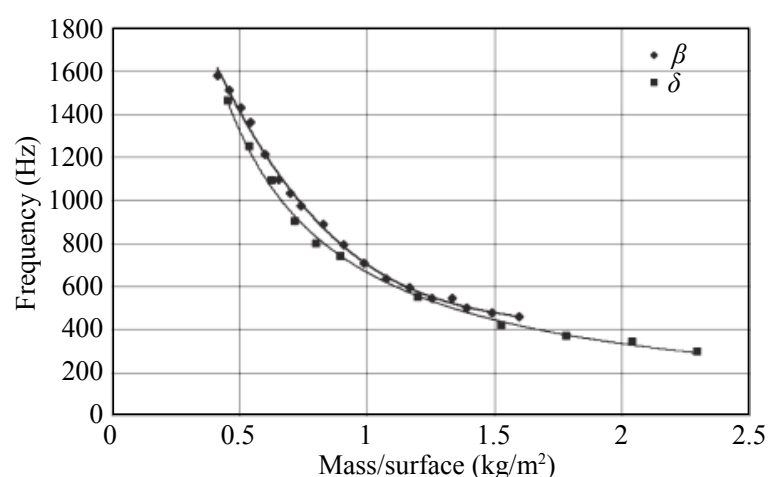

Fig. 10 Plots of the frequency of the sound absorption maximum versus the mass per unit surface of the piled disks

Table 1 Fiber diameter, density and flow resistivity of the electrospun samples

\begin{tabular}{cccc}
\hline $\begin{array}{c}\text { Fiber diameter } \\
(\mu \mathrm{m})\end{array}$ & $\begin{array}{c}\text { Density } \\
\left(\mathrm{kg} / \mathrm{m}^{3}\right)\end{array}$ & $\begin{array}{c}\text { Flow resistivity } \\
\left(\mathrm{kPa} \cdot \mathrm{s} / \mathrm{m}^{2}\right)\end{array}$ \\
\hline PVP $\beta$ & 1.6 & 63 & 440 \\
PVP $\delta$ & 2.8 & 64 & 264 \\
\hline
\end{tabular}

mechanisms. (1) Friction between the moving molecules of air and the fibers of a porous material or the internal surfaces of voids of a non-fibrous material (a dissipative absorber); (2) Energy dissipation when the sound wave is resonant with the natural frequency of vibration of a membrane; (3) Energy dissipation of a tuned cavity absorber working on the principle of a Helmholtz resonator.

The experimental results suggest that, in the present work, an important role is played by the second mechanism. Indeed, in this case, a maximum absorption is expected for the resonant frequency; this well justifies the bell-like shape of the curves. Moreover, it is well known that, whichever the geometry, a membrane has several vibration modes. This may justify the presence of a second peak so as observed for several curves in Figs. 5 and 7 -the curve $f$ of Fig. 5 and the curves e and $\mathrm{f}$ of Fig. 7. In all cases, the second maximum occurs at a frequency about 3 times greater than the one of the first peak. This result well correlates with the vibration natural frequencies spacing of circular plates which, in case of homogeneous materials, are spaced from 2 to almost 3 times the fundamental frequency, depending on the boundary conditions (from fully clamped to simply supported edges $)^{[25]}$. It is worth reminding also the data reported in Table 1 and the possible consequences - due to high flow resistivities the specimens may form a membrane-type absorber with a resonance peak at low frequencies.

Fig. 11 shows the plots, as a function of frequency, of the sound absorption coefficient altogether with the real and imaginary parts of the acoustic impedance of sample $\delta_{1-14}$. The results are representative of what happens for all the studied samples. As can be seen, the sound absorption mechanism is not purely the one of a membrane. In this case, in fact, when the sound absorption coefficient reaches the maximum, the curve of $\operatorname{Im}\left(Z / Z_{0}\right)$ should pass through the zero point ${ }^{26]}$.
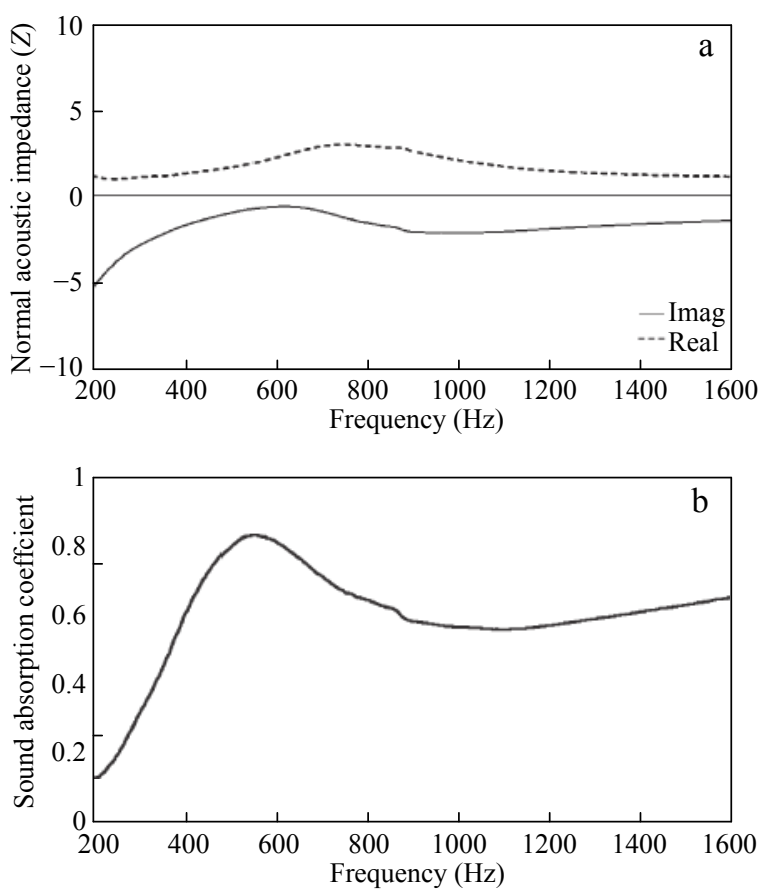

Fig. 11 Acoustic properties of PVP blanket (sample $\delta_{1-14}$ ): real and imaginary parts of acoustic impedance (a) and absorption coefficient (b)

\section{CONCLUSIONS}

Non-woven mats were successfully obtained by electrospinning PVP of high molecular weight $(1300 \mathrm{kDa})$. The mats were produced in the form of thin disks of $10 \mathrm{~cm}$ in diameter with a fiber diameter of $(1.6 \pm 0.5)$ or $(2.8 \pm$ 0.5) $\mu \mathrm{m}$. SEM observations prove that the chosen experimental conditions allow to obtain disks structurally quite homogeneous since no differences are observed between the two opposite surfaces neither from one disk to another.

The electrospun mats do possess high sound absorption coefficient in the low-medium frequency range (200$1600 \mathrm{~Hz}$ ) of interest to the transportation industry. Similar sound absorption properties are reported for microperforated panels (MPP), which, however, possess well known production technology problems. But PVP samples may have better sound absorption properties in the lower frequency range than materials of same thickness that are usually traded in both civil and aerospace engineering fields.

For a given set of disks (from a minimum of 6 ) the sound absorption coefficient changes with the frequency (in the range $200-1600 \mathrm{~Hz}$ ) following a bell shape curve with the maximum (where the coefficient is greater than 0.9) that shifts to lower frequencies with more piled disks and greater fiber diameter. The acoustic behavior can be continuously tuned by changing the mass of the blanket.

\section{ACKNOWLEDGMENTS}

This work has been partially supported and funded by the Department of Education, Research, Labour, of the Cultural Politics and Social Politics of Campania Region under the research program 
"MITO-Improvement and Innovation of "Thermoacoustical Material for Aeronautical Applications" PO FESR Campania 2007/2013, OO 2.1; CUP: B68C12000640007, Code SMILE: 150. This support is greatly appreciated and acknowledged by the authors. Professor Gino Iannace of the Dept. of "Architettura e Disegno Industriale" of the University of Campania "Luigi Vanvitelli", member of UNI committee on Acoustics and Vibration, who kindly made available the apparatus for the measurement of flow resistivity, is highly acknowledged. The valuable experimental support of Luciano Cortese, in charge of SEM laboratory of Istituto di Ricerche sulla Combustione of Consiglio Nazionale delle Ricerche of Naples, is highly acknowledged.

Open Access This article is distributed under the terms of the Creative Commons Attribution License which permits any use, distribution, and reproduction in any medium, provided the original author(s) and the source are credited (http://creativecommons.org/ leicenses/by/4.0/).

\section{REFERENCES}

1 Barber, A. "Handbook of noise and vibration control", Elsevier, Oxford, 1992

2 Crocker, M. J. "Handbook of noise and vibration control", John Wiley and Sons, New York, 2007

3 Khan, W. S.; Asmalutu, R.; Yildirim, M. B. Acoustical properties of electrospun fibers for aircraft interior noise reduction. J. Aerosp. Eng. 2012, 25, 376-382.

4 Ingard, U. Notes on sound absorption technology. 1994

5 Goines, L.; Hagler, L. Noise Pollution: A Modern Plague. South. Med. J. 2007, 100, 287-294.

6 Mahashabde, A.; Wolfe, P.; Ashok, A.; Dorbia, C.; He, Q.; Fan, A.; Lukachko, S.; Mozdzanowska, A.; Wollersheim, C.; Barrett, S. R. H.; Locke, M.; Waits, I. A. Assessing the environmental impacts of aircraft noise and emissions. Prog. Aerosp. Sci. 2011, 47, 15-52.

7 Zhao, D.; Li, X. Y. A review of acoustic dampers applied to combustion chambers in aerospace industry. Prog. Aerosp. Sci. 2015, 74, 114-30.

8 Harris, C. M., "Handbook of Acoustical Measurements and Noise Control", Mcgraw-Hill, 1997

9 Arenas, J. P.; Crocker, M. J. Recent trends in porous soundabsorbing materials. J. Sound Vib. 2010, 12-17.

10 Liu, H.; Wang, D.; Zhao, N.; Ma, J.; Gong, J.; Yang, S.; Xu, J. Application of electrospinning fibres on sound absorption in low and medium frequency range. Mater. Res. Innov. 2014, 18, 888-891.
11 Huang, Z. M.; Zhang, Y. Z.; Kotaki, M.; Ramakrishna, S. A review on polymer nanofibers by electrospinning and their applications in nanocomposites. Compos. Sci. Technol. 2003, 63, 2223-2253.

12 Teo, W. E.; Ramakrishna, S. A review on electrospinning design and nanofibre assemblies. Nanotechnology 2006, 17, 89-106.

13 Rutledge, G. C.; Fridrikh, S. V. Formation of fibers by electrospinning. Adv. Drug Deliv. Rev. 2007, 59, 1384-1391.

14 Bhardwaj, N.; Kundu, S. C. Electrospinning: A fascinating fiber fabrication technique. Biotechnol. Adv. 2010, 28, 325-347.

15 Lanotte, L.; Bilotti, C.; Sabetta, L.; Tomaiuolo, G.; Guido, S. Dispersion of sepiolite rods in nano fibers by electrospinning. Polymer 2013, 54(4), 1295-1297.

16 Agarwal, S.; Greiner, A.; Wendorff, J. H. Functional materials by electrospinning of polymers. Prog. Polym. Sci. 2013, 38, 963-991.

17 Xiang, H.; Tan, S.; Yu, X.; Long, Y.; Zhang, X.; Zhao, N.; Xu, J. Sound absorption behavior of electrospun polyacrylonitrile nanofibrous membranes. Chinese J. Polym. Sci. 2011, 29(6), $650-657$.

18 Trematerra, A.; Iannace, G.; Nesti, S.; Fatarella, E.; Peruzzi, F. Acoustic properties of nanofibers. Noise \& Vibration Worldwide 2014, 45, 29-33.

19 Iannace, G. Sound absorption of materials obtained from the shredding of worn tyres. Building acoustics 2014, 21(4), 277-286.

20 Chung, J. Y.; Blaser, D. A. Transfer function method of measuring in-duct acoustic properties. I. Theory. J. Acoust. Soc. Am. 1980, 68, 907-13.

21 Koruk, H. An assessment of the performance of impedance tube method. Noise Contr. Eng. J. 2014, 62, 264-274.

22 Shenoy, S. L.; Bates, W. D.; Frisch, H. L.; Wrek, G. E. Role of chain entanglements on fiber formation during electrospinning of polymer solutions: Good solvent, non-specific polymerpolymer interaction limit. Polymer 2005, 46, 3372-3384.

23 Tarnow, V. Measured anisotropic air flow resistivity and sound attenuation of glass wool. J. Acoust. Soc. Am. 2002, 111(6), $2735-2739$.

24 Stani, M. M.; Muellner, H.; Plotizin, I. Sound insulation of plasterboard walls and air flow resistivity: an empirical examination with respect to practical applications. Proceedings of forum acusticum 2005 Budapest, 1987-1992

25 Blevins, R. D. Formulas for natural frequency and mode shape. Krieger Pub Co, ISBN-13: 978-1575241845, ISBN-10: 1575241846, 2001

26 Zhu, X. Z.; Chen, Z. B.; Jiao, Y. H.; Wang, Y. P. Broadening of the sound absorption bandwidth of the perforated panel using a membrane-type resonator. ASME J. Vib. Acoust. 2018, 140(3), 031014 . 